\title{
Síndrome de burnout en el personal de salud de las áreas de cirugía general y emergencia del Hospital General Puyo
}

Burnout syndrome in the health personnel of the general and emergency surgery areas of the General Puyo Hospital

María José Ortiz Rojas. ${ }^{1}$, Karina Vanessa Ortiz Jácome. ${ }^{2}$ \& Carlos Xavier Haro Erazo. ${ }^{3}$

Recibido: 17-06-2019 / Revisado: 21-07-209 /Aceptado: 14-08-2019/ Publicado: 06-09-2019

\section{Abstract DOI: https://doi.org/10.33262/cienciadigital.v3i3.3.822}

Burnout refers to the loss of professional responsibility and when talking about this syndrome, the following triad must be taken consider: emotional exhaustion, depersonalization and diminished sense of accomplishment. (1)

The Burnout syndrome began to be analyzed from the 70 s due to the worldwide transformation in the field of work (2). Burnout or work stress is related to those professionals who have direct contact with other professions such as doctors and nurses.

The burn syndrome has different components that negatively impact the personal and service context, resulting in a higher rate of absenteeism and work demotivation, as well as a decrease in the quality of care of the institutions. (3)

Given this reality, this work is carried out to determine the situational diagnostic of this syndrome in personnel working at Emergency and General Surgery areas of the General Hospital of Puyo, so that preventive measures are taken, in order to ensure for the integral health of workers. For the diagnosis, the Maslach test was applied, from a total

\footnotetext{
1 Universidad Nacional de Chimborazo,majosortiz57@homtail.com

2 Universidad Nacional de Chimborazo, karinita_1106@hotmail.com

3 Universidad Central del Ecuador, carlox.chex87@hotmail.com
} 
of 120 professionals.

Keywords: Burnout, work stress, emotional fatigue, depersonalization, burn syndrome.

\section{Resumen}

Burnout hace referencia a la pérdida de responsabilidad profesional y por ende al hablar de este síndrome se debe tomar en cuenta la siguiente triada: agotamiento emocional, despersonalización y sentido de realización disminuido. (1)

El síndrome de Burnout empezó a ser analizado a partir de los años 70 debido a la transformación a nivel mundial en el ámbito del trabajo (2). El Burnout o estrés laboral se relaciona con aquellos profesionales que tienen contacto directo con otras profesiones como es el caso de médicos y enfermeras. (3)

El síndrome del quemado posee diferentes componentes que impactan negativamente en el contexto personal y de servicio, se traduce en un mayor índice de ausentismo y desmotivación laboral, además de una disminución en la calidad de atención de las instituciones. (3)

Ante esta realidad, se realiza este trabajo para determinar el diagnóstico situacional de este síndrome en el personal que labora en las áreas de Emergencia y Cirugía General del Hospital General del Puyo, de tal manera que se tomen las medidas preventivas, con la finalidad de velar por la salud integral de los trabajadores. Para el diagnóstico se aplicó el test de Maslach, de una muestra total de 120 profesionales.

Palabras claves: Burnout, estrés laboral, cansancio emocional, despersonalización, síndrome del quemado.

\section{Introducción}

A partir de 1976 Maslach uso el término "burnout", empleado por abogados de California para describir el proceso gradual de pérdida de responsabilidad profesional y desinterés entre compañeros de trabajo, para hacer alusión a un conjunto de respuestas emocionales que afectaban a los profesionales. (1) 
El síndrome de burnout está caracterizado por la presencia de la siguiente triada, la cual la conforman: 1) agotamiento emocional, 2) despersonalización y 3) sentido de realización disminuido. Las manifestaciones pueden llegar a un cuidado subóptimo de los pacientes y descuido de la salud física y mental del profesional; se usa como método de reconocimiento la Escala de Maslach.

El burnout es definido inicialmente por Maslach y Jackson desde 1981 como un síndrome de carácter psicológico de agotamiento emocional, agotamiento de los propios recursos emocionales y la presencia de sentimientos de agotamiento emocional y psicológico. Es decir, resulta de una respuesta prolongada a los factores estresores interpersonales de tipo crónico obtenidos dentro del ámbito laboral y tiende a transformarse de forma estable con el tiempo. Se añade su presencia como el resultado del ambiente social en el cual trabajan según las condiciones laborales en las cuales no se reconoce el lado humano del trabajo, se puede llegar a generar una brecha muy amplia entre la naturaleza del trabajo y la de las personas, lo cual ocasiona un mayor riesgo de desarrollo del presente síndrome. (2)

El burnout o estrés laboral asistencial según Moreno, Oliver y Aragoneses (1991, p. 271) "hace alusión a una clase de estrés laboral, ocasionado especialmente en aquellas profesiones en las que existe contacto directo con las personas usuarias, más concretamente, podemos hablar de profesiones que prestan ayudas tales como: médicos, enfermeras, maestros, policías". Es así que una actual visión del estrés laboral se ha concentrado con mayor énfasis quienes son prestadores de servicios de salud, siendo este el más prevalente; se han señalado tres grupos principales de factores socio laborales que resultan muy significativos para la salud médica: 1) aumento de pacientes en la atención médica, cuyo resultado es el aumento del estrés tanto emocional y mental; 2) aumento de los requerimientos institucionales, que exige mayor adaptación de los trabajadores a las condiciones de cada servicio que se oferte y 3) pobres condiciones laborales, sumadas al incremento del número de horas laborales, desempleo eventual, inestabilidad laboral, entre otros. Con lo mencionado, aumenta la prevalencia del desgaste profesional en el campo médico (4) 
La Organización Mundial de la Salud (OMS) menciona: "que la sobrecarga laboral afecta en forma negativa a la salud mental y física de los empleadores, disminuyendo su calidad de vida y a la eficacia de las entidades para las que laboran" (3)

El desgaste profesional; a nivel presenta una alta prevalencia en varios estudios: en España, en un hospital de Madrid se evidenció $14 \%$ dentro del personal de salud, con mayor frecuencia en médicos. Altos niveles de burnout se encontraron en estudios realizados en Francia con una prevalencia de $46.5 \%$. En la actualidad existen estudios en todo el mundo como Japón, EEUU y Serbia entorno al síndrome de burnout y factores asociados (3).

A nivel de Latinoamérica, este síndrome se encuentra presente en los profesionales de la salud de: Perú, Ecuador, México, Colombia, Guatemala y El Salvador presentaron incidencias que oscilan de $2,5 \%-5,9 \%$, con predominio en la carrera de medicina con una prevalencia del $12,1 \%$, seguida de enfermería con $7,2 \%$, las otras profesiones dentro del área de salud presentaron una prevalencia de menos del 6\%. Se encontró incluso una alta prevalencia de este síndrome en los médicos que laboran en el área de urgencias (17\%) y médicos internistas $(15,5 \%)$, en contraposición a las especialidades de dermatología y anestesiología que obtuvieron las prevalencias más bajas 5,3\% y 5\%, respectivamente. (3) El estrés resulta de un proceso psicofisiológico que se desencadena por una situación laboral determinada que provoca un desequilibrio significativo entre la demanda que experimenta un sujeto y los recursos adaptativos de que dispone (5)

Según estudios epistemológicos el personal del área de enfermería se encuentra afectado en un 25\% (Demeuroti \& Bakker, 2000); en el año 2013 según un estudio llevado a cabo en la ciudad de Quito entre junio a diciembre de 2013, el mismo que tuvo una duración de 6 meses; encontró que entre el $6-46 \%$ del personal profesional de enfermería presento una baja realización personal (6).

La investigación de síndrome de Burnout implica la relación con otras variables que son altamente relevantes como es: calidad de vida profesional, respecto a lo cual existen posturas teórico-metodológicas que sustituyen indistintamente el término calidad de vida en 
el trabajo, por calidad de vida laboral y/o profesional (CVP) (Rosas, Preciado, Plascencia, \& Colunga, 2016); la cual se puede entender como el grado de percepción del trabajador de sus necesidades personales (Gonzáles, Hidalgo Salazar, \& Preciado, 2010).

Es así que resulta altamente imperioso considerar a la calidad de vida profesional como el grado de satisfacción personal y profesional existente en el puesto de trabajo, su desempeño y en el ambiente laboral, que viene dado por cierto tipo de dirección y gestión, condiciones de trabajo, compensaciones, atracción e interés por las actividades realizadas; y nivel de logro y autodesarrollo (9)

El agotamiento tiene relaciones muy estrechas con los resultados en el lugar de trabajo, incluyendo dentro de ellos los problemas de salud y déficit de desempeño. Las intervenciones han explorado diversas formas de programas para lograr mejorar o prevenir el grado de agotamiento por parte del profesional. Las diferentes iniciativas creadas para mejorar la calidad de los entornos sociales en el lugar de trabajo han mostrado resultados muy alentadores debido a los casos antes planteados, $\mathrm{y}$, al rápido avance con el que Burnout o estrés laboral empieza a desarrollarse, sin tomar en cuenta el estatus social, cultural o tradiciones, es por ello que se decide el empezar a establecer distintos factores para evitar que esta condición se convierta un problema de salud pública en Ecuador.

Con el presente trabajo pretendemos demostrar que dentro del Área de Emergencia y Cirugía General existen altas cifras de Síndrome de Burnout en el Hospital General del Puyo. A su vez dentro de los objetivos principales que se persigue con esta investigación es conocer el índice de Burnout dentro del personal sanitario que labora en el Área de Emergencia y Cirugía General; establecer cuál es el componente de la triada de Burnout que se encuentra presente dentro del personal sanitario que labora en el Área de Emergencia y Cirugía General.

\section{Importancia del problema}

La pérdida de motivación o incentivo, especialmente donde la dedicación a una causa no produce los resultados deseados, resulta en un alto grado de estrés de las personas que laboran en carreras de salud, como médicos y enfermeras, que prestan servicio a cambio de 
un salario o una formación profesional (2)

Desde una perspectiva médica se ha visto que el alto grado de estrés laboral, una inadecuada relación y comunicación médico paciente basados en los tiempos promedio de atención sobre todo en sector público entre otros son factores que han desencadenado las constantes características de un síndrome de burnout por lo que planteamos siempre la necesidad de realizar valoraciones por parte de los servicios de medicina ocupacional de las instituciones en el personal de salud de manera periódica. $(4,7,9)$

\section{Metodología}

Se realiza un estudio de tipo descriptivo - transversal, con un total de 120 trabajadores de la salud, pertenecientes al Hospital General Puyo, que comparten ciertas características comunes, se pretende establecer la prevalencia del síndrome de Burnout en los participantes. Se realiza un cuestionario individual de variables sociodemográficas y laborales y una evaluación específica con la escala de Maslach Burnout Inventory (MBI), que plantea 22 enunciados sobre sus sentimientos y pensamientos en relación a las actividades realizadas en el trabajo.

Existen 3 sub escalas dentro de la escala MBI

1. Agotamiento emocional (AE) (emotional exhaustion), 9 ítems que describen sentimientos de estar abrumado y agotado emocionalmente por el trabajo; fatiga, que aparecen como consecuencia de la actividad laboral. La falta de energía y destrucción de sus recursos emocionales y no queda nada que dar u ofrecer a los demás surgiendo ansiedad, impaciencia, irritabilidad y actitudes suspicaces. Corresponde a los ítems 1, 2, 3, 6, 8, 13, $14,16,20$.

2. Despersonalización (D) (despersonalization), 5 ítems que evalúan la despersonalización y negatividad hacia los usuarios. Se define como la pérdida de autoestima, conceptos y actitudes negativas e incapacidad para afrontar situaciones que antes lo hacía con eficacia. Corresponde a los ítems 5,10,11,15,22. 
3. Realización personal en el trabajo (RP) o Reducido logro personal (personal accomplishment), 8 ítems que evalúan la progresiva sensación de incompetencia de la persona y su imposibilidad de satisfacer las expectativas de su profesión. Corresponde a los ítems 4, 7, 9,12, 17, 18, 19,21.

Se establecerá la significancia del este estudio, asignando un valor de $\mathrm{p}=0,05$

\section{Resultados}

- Del análisis de las variables sociodemográficas del total de 120 individuos estudiados $42,5 \%$ pertenecen al sexo femenino y $57,5 \%$ al sexo masculino, el $45 \%$ de los estudiados tienen una edad que oscila entre 31 a 40 años, $30.8 \%$ entre 21 a 30 años.

- En cuanto al estado civil el 53\% refiere estado civil casado, y con dependencia económica de 1 a 2 hijos el $55.8 \%$

- El 90\% de los encuestados niega tener hábitos de alcoholismo o tabaquismo.

- De los estudiados el $45 \%$ son médicos, $25 \%$ son enfermeras y $30 \%$ auxiliares de enfermería.

- En lo que se refiere a años de profesión existe un total de 28 médicos, 9 enfermeras y 9 auxiliares de enfermería que laboran de 1 a 5 años, 41 personas que tienen de 6 a 10 años de profesión y 33 labora más de 10 años en la institución, lo que indica que el 72,5\% de los estudiados trabajan menos de 10 años en la institución, por lo tanto, resulta ser una población joven.

- El $86,7 \%$ de los encuestados indica presentar gusto por su trabajo, 55,8\% refieren que existe autoexigencia y exigencia hacia lo demás, $20 \%$ expresa que siempre se reconoce su trabajo y a veces un $51,7 \%$.

- La capacitación y motivación hacia los trabajadores es permanente de un 33\% de los encuestados y evaces en un $35,8 \%$ 
Tabla 1. Area de Trabajo

\begin{tabular}{llc}
\hline \multicolumn{1}{c}{ Areas de Trabajo } & Fr & $\%$ \\
\hline CIRUGÍA GENERAL & 38 & 31.7 \\
EMERGENCIA & 82 & 68.3 \\
\hline
\end{tabular}

Fuente: Cuestionario variables sociodemográficas y laborales

Elaboración: Autores

Análisis: del total de encuestados la mayoría labora en el área de emergencia, representado en un $82 \%$ de los encuestados.

Tabla 2. Horas diarias de trabajo

\begin{tabular}{lcc}
\hline Horas diarias de trabajo & Fr & $\%$ \\
\hline 4 & 0 & 0 \\
6 & 6 & 5 \\
8 & 52 & 43.33 \\
Horario Rotativo & 61 & 51.67 \\
\hline
\end{tabular}

Fuente: Cuestionario variables sociodemográficas y laborales

Elaboración: Autores

Análisis: 51,67 \% de los trabajadores labora en horarios rotativos, seguido del $43.44 \%$ que laboran en un periodo de 8 horas.

Tabla 3. Control en los horarios

\begin{tabular}{lcc}
\hline Problemas & Fr & $\%$ \\
\hline Exceso de pacientes & 21 & 17,6 \\
Falta de material & 45 & 37,5 \\
Falta de apoyo & 38 & 31,66 \\
Falta de exigencia de las autoridades & 16 & 13,24 \\
\hline
\end{tabular}

Fuente: Cuestionario variables sociodemográficas y laborales

Elaboración: Autores

Análisis: la mayoría de los trabajadores en un 37.5\% refiere que el problema principal en cuanto a pérdida de control, es la falta de material en la institución. 
Tabla 4. Relación de edad, profesión y formación académica con factores laborales

\begin{tabular}{lccc}
\hline \multicolumn{1}{c}{ Categortización } & Edad & Profesión & $\begin{array}{c}\text { Formación } \\
\text { Académica }\end{array}$ \\
\hline Estabilidad laboral & 0 & - & - \\
Ambiente trabajo & 0 & - & - \\
Atención enfermos diarios & - & - & 0,001 \\
Exigente con usted y los que rodea & - & 0,002 & - \\
Dependencia económica & 0 & - & - \\
Horas diarias trabajo &, $002-$ & - & - \\
Años profesion & 0 & 0,001 & 0 \\
Limitación técnica económica & - & 0,004 & 0,001 \\
Lugar de trabajo & - & 0,004 & 0,002 \\
Capacitación & - & & \\
$* *$ p menor 0.05 & & & \\
\hline
\end{tabular}

Fuente: Cuestionario variables sociodemográficas y laborales

Elaboración: Autores

Análisis: p estadísticamente significativas con un valor menor de 0.05

TABLA 5. Relación de preguntas de maslash con factores socio demogragicos.

\begin{tabular}{|c|c|c|c|c|c|c|c|c|c|c|c|c|c|c|c|c|c|}
\hline & $\begin{array}{l}\text { से } \\
\text { t. }\end{array}$ & 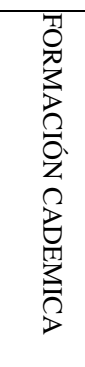 & 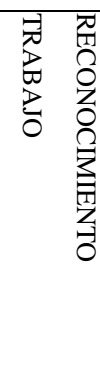 & 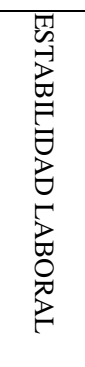 & 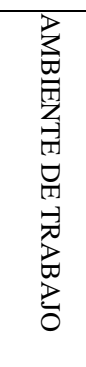 & 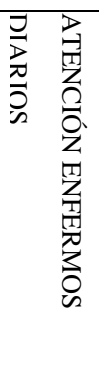 & 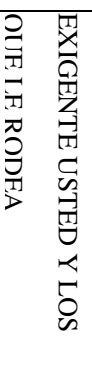 & 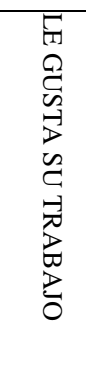 & 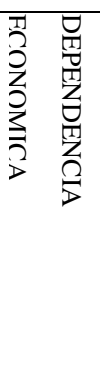 & 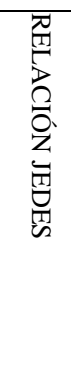 & 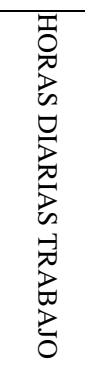 & $\begin{array}{l}3 \\
z \\
0 \\
0 \\
0 \\
0 \\
0 \\
0 \\
17 \\
0 \\
0 \\
z \\
z\end{array}$ & 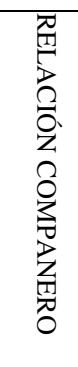 & 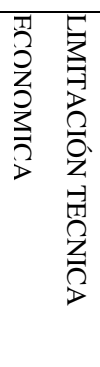 & 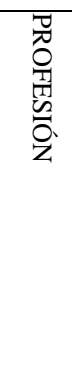 & 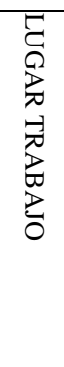 & $\begin{array}{l}z \\
z \\
0 \\
0 \\
0 \\
0 \\
\Delta \\
0 \\
D \\
0\end{array}$ \\
\hline P1 & - & 0,032 & 0,004 & 0 & 0,025 & - & - & - & - & - & - & - & - & - & - & - & \\
\hline P2 & - & 0,006 & - & - & - & 0,021 & 0,044 & 0,005 & 0,003 & - & - & - & - & - & - & - & \\
\hline P3 & - & 0,004 & - & - & - & 0,021 & - & - & $=$ & - & - & - & - & - & - & - & \\
\hline P4 & - & ,022- & - & - & - & - & - & - & - & - & - & - & - & - & - & - & \\
\hline P5 & - & - & - & - & - & - & - & - & - & - & 0,039 & - & - & - & - & - & \\
\hline P6 & - & - & - & - & - & - & - & - & - & - & - & - & - & - & - & - & \\
\hline P7 & - & - & - & - & - & - & - & - & - & - & - & - & - & - & - & - & \\
\hline P8 & - & - & - & - & - & 0,032 & - & 0,002 & - & - & 0,033 & 0,02 & 0,022 & - & - & - & \\
\hline P9 & - & - & - & 0,002 & - & - & - & - & - & - & - & - & - & 0,035 & - & - & \\
\hline P10 & - & - & - & - & - & - & - & - & - & - & - & - & - & - & 0,03 & - & \\
\hline P11 & - & - & - & - & - & - & - & 15 & - & - & - & 0,02 & - & - & 0,04 & - & \\
\hline P12 & 0 & - & - & - & 0,001 & - & - & 0,01 & - & - & - & - & - & - & 0,04 & - & \\
\hline
\end{tabular}


TABLA 5. Relación de preguntas de maslash con factores socio demogragicos (Continuación)

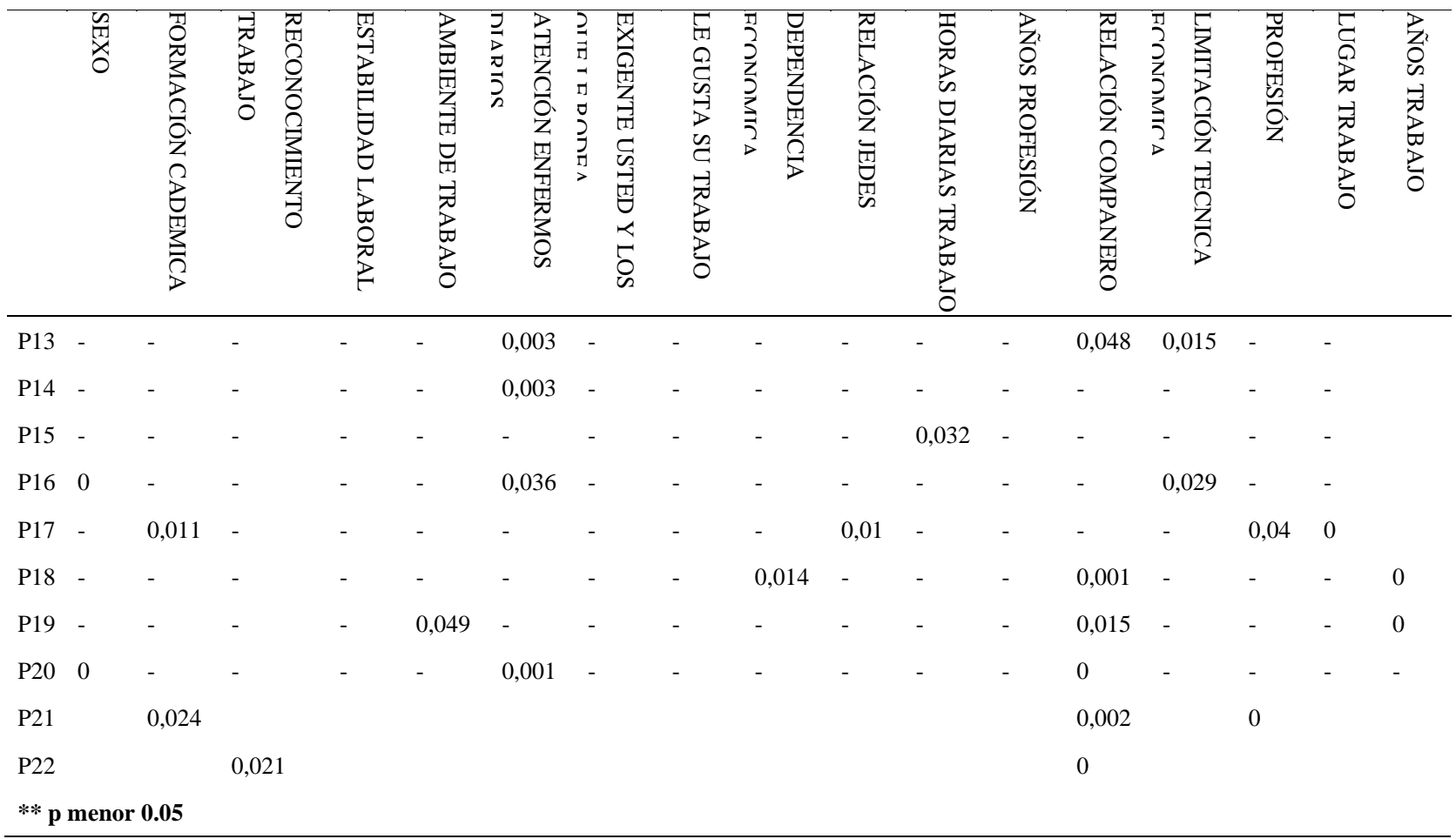

Fuente: Cuestionario variables sociodemográficas y laborales, Escala de Maslach Burnout Inventory (MBI)

Elaboración: Autores

ANÁLISIS: p estadísticamente significativas con un valor menor de 0.05

Figura 1. Diagnóstico del síndrome de burnout, hospital general Puyo junio 2019

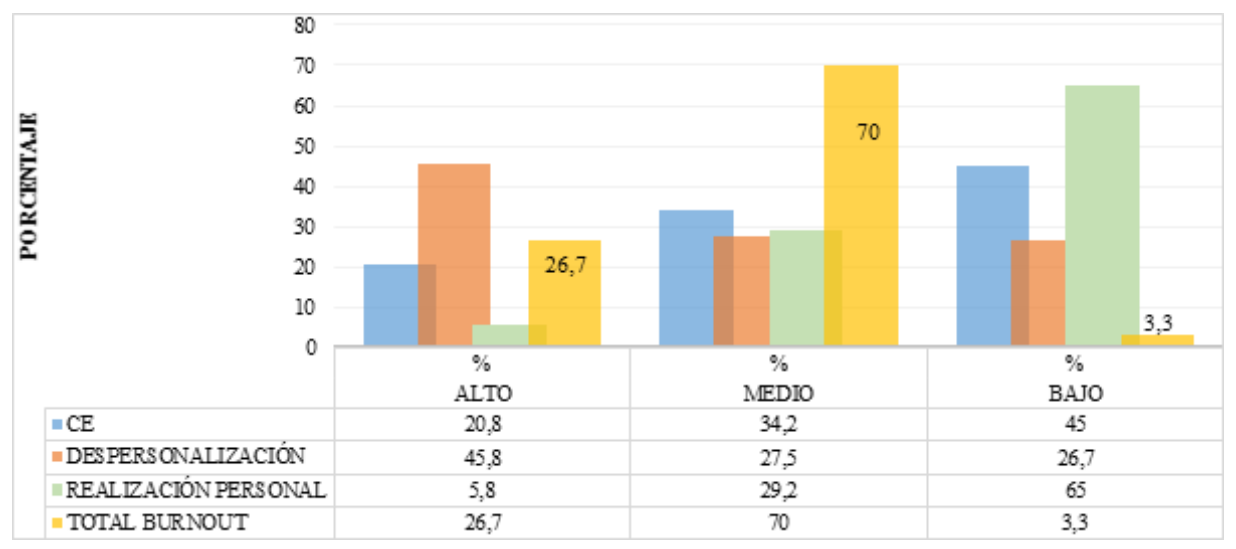

Fuente: Escala de Maslach Burnout Inventory (MBI)

Elaboración: Autores 
Análisis: $70 \%$ de los trabajadores encuestados en el Hospital General Puyo, después de su respectivo análisis presentan un nivel medio de Síndrome de Burnout.

\section{Discusión}

En el siglo XXI, la investigación sobre el burnout se ha ido consolidando en Latinoamérica y se le da reconocimiento como un problema de salud vinculado a los riesgos psicosociales en y del trabajo (Gil-Monte, 2007). Se considera que el estudio del burnout ha avanzado en la medida en que se considera como una problemática de salud que se presenta en el contexto laboral (2), nuestro estudio corrobora la presencia de un nivel medio de síndrome de burnout. La investigación realizada en nuestro estudio nos permitió determinar la presencia en médicos enfermeras del síndrome de burnout como otros estudios ya realizados sin embargo creemos que se debería hacer seguimiento y tomar medidas correctivas para este problema de salud evidente en las áreas de salud de numerosas instituciones de salud a nivel mundial razón por la cual creemos necesario realizar comparaciones con estudios posteriores.

\section{Conclusiones}

- Existe gran porcentaje del personal de salud estudiado que presenta síndrome de Burnout en un nivel medio, en el Hospital General Puyo.

- La mayoría de encuestados son mujeres, y la edad de la mayoría de los entrevistados oscila entre 31 a 40 años, por lo que la prevalencia de Burnout en este estudio, resulta ser mayor en estas poblaciones.

- De los profesionales de la salud estudiados, la mayoría resultan ser médicos, por lo que el riesgo de sufrir síndrome Bournout en este estudio se ve aumentado en el ejercicio de esta profesión.

- La satisfacción laboral predomina en los resultados, sin embargo, existen situaciones como la falta de material, la exigencia por parte del ámbito superior, la falta de capacitación y motivación, que resultan un detonante para la aparición de estrés y frustración en los trabajadores. 
- La falta de reconocimiento laboral por parte de las jefaturas, según refieren los entrevistados, es un factor que aumenta el nivel de motivación en su trabajo, lo que provoca que sus actividades en ocasiones sean realizadas sin el interés debido, esto genera inestabilidad laboral y su vez mayor preocupación y estrés, situación que puede ser analizada y corregida.

- Las áreas críticas de salud, específicamente en este caso el área de Emergencia y el área quirúrgica, son áreas que, por su exigencia, atención masiva de pacientes, gravedad de los mismos y otros factores, resultan ser zonas de riesgo para la aparición de síndrome de Bournot en los profesionales

\section{Referencias}

1. Carrillo LD, Carrillo JR, Carrillo R, Garduño ML. Prevalencia de depresión y síndrome de burnout en residentes de urología de un hospital de tercer nivel. Rev $\begin{array}{lllll}\text { Mex Urol. } 2018 \text { marzo-abril; } 78 & \text { (2):105-111. Doi: }\end{array}$ https://doi.org/10.24245/revmexurol.v78i2.1492

2. Díaz F, Gómez I. Revista Sicología desde el Caribe. La investigación sobre el síndrome de burnout en Latinoamérica entre 2000 y 2010, Volumen 33 Numero 1, enero-abril 2016. Disponible en: http://dx.doi.org/10.14482/psdc.33.1.8065

3. Flores J. Prevalencia y Factores asociados al síndrome de Burnout en Médicos asistentes del servicio de emergencia del hospital Marino Molina Sccipa. Septiembre - noviembre 2017. Lima-Perú. Disponible en: http://repositorio.urp.edu.pe/handle/urp/1153

4. Gutiérrez O, Loboa N, Martínez J. Prevalencia del Síndrome de Burnout en profesionales de enfermería de la Orinoquia colombiana. 2016. Revista Universidad y Salud. Universidad de los llanos. Villavicencio, Colombia. Doi: https://doi.org/10.22267/rus.182001.107

5. Hernández G. Burnout en Médicos de un hospital del sector público en el Estado de Hidalgo. Estado de Hidalgo junio 2018. Revista Scielo. Disponible en: 
<http://www.scielo.org.mx/scielo.php?script=sci_arttext\&pid=S187054722018000200161\&lng=es\&nrm=iso >. ISSN 1870-5472.

6. Mariño C, Astrid C. Burnout en profesionales de la salud en Ecuador - Quito 2018. Trabajo de Titulación de Licenciado en Psicología. UTPL, Quito. Disponible en: http://dspace.utpl.edu.ec/handle/20.500.11962/22382

7. Muñoz S, Ordoñez J, Solarte M, Valverde Y, Villareal S. Síndrome de Burnout en enfermeros del hospital Universitario San José. Popayán. Enero 2018. Rev. Méd. Risaralda 2018; 23 (1): 34 - 37. Disponible:

http://www.scielo.org.co/scielo.php?script=sci_arttext\&pid=S012206672018000100006

8. Oliva GJ. Síndrome de Burnout y resiliencia en el personal de salud del Hospital María Auxiliadora. Lima 2017. Trabajo para la obtención de tirulo de Médico Cirujano. Disponible en http://repositorio.urp.edu.pe/handle/URP/1181

9. Preciado DA, Herrera JA, Gómez J, Cortés P, Ortega M. Niveles de estrés y síndrome de burnout en urólogos mexicanos. ¿Cómo nos encontramos? Rev Mex Urol. 2018 marzo-abril; 78(2):98-104. Doi: https://doi.org/10.24245/revmexurol.v78i2.1741.

10. Unamuno NR. Análisis de los factores que inciden en el síndrome de Burnout en los empleados de las fiduciarias de la ciudad de Guayaquil. Guayaquil 2018. Trabajo previo a la obtención del grado académico: MAGISTER EN ADMINISTRACIÓN DE EMPRESAS.

Disponible

en http://repositorio.ucsg.edu.ec/bitstream/3317/10922/1/T-UCSG-POS-MAE-180.pdf

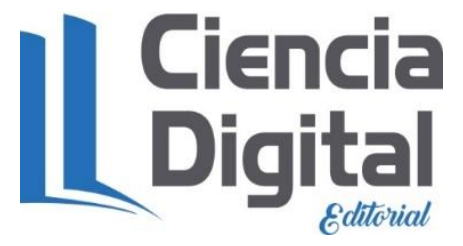


PARA CITAR EL ARTÍCULO INDEXADO.

Ortiz Rojas, M., Ortiz Jácome, K., \& Haro Erazo, C. (2019). Síndrome de burnout en el personal de salud de las áreas de cirugía general y emergencia del Hospital General Puyo. Ciencia Digital, 3(3.3), 295-308. https://doi.org/10.33262/cienciadigital.v3i3.3.822

\section{Ciencia \\ Digital}

El artículo que se publica es de exclusiva responsabilidad de los autores y no necesariamente reflejan el pensamiento de la Revista Ciencia Digital.

El artículo queda en propiedad de la revista y, por tanto, su publicación parcial y/o total en otro medio tiene que ser autorizado por el director de la Revista Ciencia Digital.
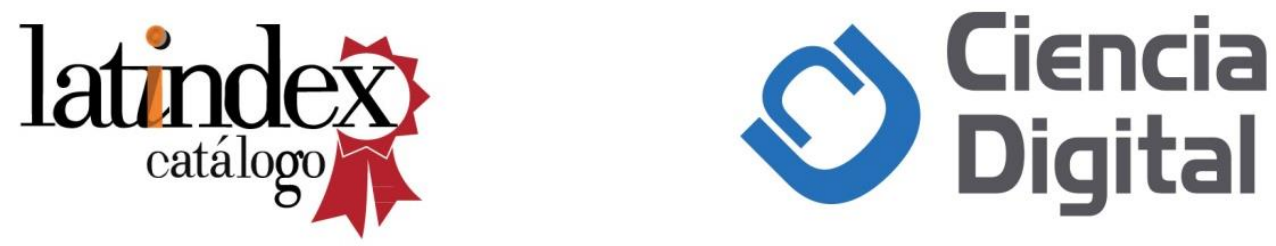